Anal ysi s of sat urat i on phenomena in Cer enkov free- el ectron I asers wi th a pl anar wavegui de

\begin{tabular}{|l|l|}
\hline 著者 & Far es Hesham Yamada M nor u \\
\hline $\begin{array}{l}\mathrm{j} \text { our nal or } \\
\text { publ i cat i on ti t l e }\end{array}$ & Physi cs of Pl asmas \\
\hline vol une & 18 \\
\hline number & 9 \\
\hline page r ange & 93106 \\
\hline year & 2011- 09-01 \\
\hline URL & ht t p: //hdl . handl e. net /2297/29455 \\
\hline
\end{tabular}




\title{
Analysis of saturation phenomena in Cerenkov free-electron lasers with a planar waveguide
}

\author{
Hesham Fares ${ }^{1,2, a)}$ and Minoru Yamada ${ }^{1}$ \\ ${ }^{1}$ Division of Electrical Engineering and Computer Science, Kanazawa University, \\ Kakuma-machi, Kanazawa, Ishikawa 920-1192, Japan \\ ${ }^{2}$ Department of Physics, Faculty of Science, Assiut University, Assiut 71516, Egypt
}

(Received 12 April 2011; accepted 10 August 2011; published online 9 September 2011)

\begin{abstract}
In this paper, the operation of the stimulated emission in Cerenkov free-electron laser (CFEL) is studied on the basis of the modulations of electron velocity and density by the electromagnetic (EM) field. The influence of the electron relaxation, due to mutual electrons collisions, on the electron dynamics is taken into account. We investigate the growth characteristics of Cerenkov laser operating in the small-signal and saturation regimes. In the saturation regime, the effect of velocity reduction of the electron beam on the gain dynamics is demonstrated. We also show that our results match with those of other well-known treatments in the small-signal gain limit. (C) 2011 American Institute of Physics. [doi:10.1063/1.3630942]
\end{abstract}

\section{INTRODUCTION}

The Cerenkov free-electron laser (CFEL) is of high interest to produce tunable radiation in the microwave ${ }^{1-10}$ and far-infrared ranges ${ }^{11-15}$ or even in an optical wavelength range. ${ }^{16-21}$ In the CFEL, an electron beam moves along a surface of a dielectric medium by which the propagating velocity of an electromagnetic wave (EM-wave) is slowed down. The electron beam can transfer its energy to the EM wave when the electron beam velocity matches with the phase velocity of the EM wave. Downsizing of the scale of the electron accelerators is essential for the compactness of the CFEL operated with a moderate acceleration voltage (below $100 \mathrm{kV}$ ).

In a previous paper, ${ }^{22}$ the present author introduced a unified analysis to describe the spontaneous and stimulated emissions of the CFEL operation on the basis of the single electron model in the small-signal regime. The dynamics of the EM wave were described by the Maxwell's wave equation which includes the exciting current of electron beam; thus, the spontaneous and stimulated emissions were investigated basing on the utilized formulations of the electron beam current. For the spontaneous emission, the electron beam current was expressed as a sum of irregularly distributed electrons. For the stimulated emission, the electron beam current was described corresponding to the modulations of the electron velocity and density by the EM wave. In these analyses, the average drift velocity of the electron beam is approximated to be constant.

The dynamics of velocity and density modulations should be influenced by the mechanism of so-called electron relaxations resulting from the Coulomb scatterings "collisions" among electrons. ${ }^{22-24}$ Initially, when the force induced from the electric field starts to bunch the moving electrons, dense and sparse parts in the electrons distribution appear alternately. The asymmetric Coulomb forces exerted on an electron by its neighbor electrons will act as damping

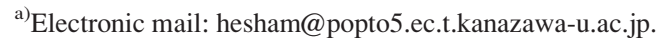

forces for modulations of electron density and velocity. The damping mechanism characterized by the so-called the relaxation time works to attenuate the modulated component toward the average value. ${ }^{22,25-27}$

This paper aims at the saturation analysis of the interaction between the EM wave and the electron beam in the CFELs device. There have been many studies for the CFELs operated in the nonlinear saturation state. ${ }^{28-32}$ To analyze the saturation phenomena in the CFEL operation, the attenuation effect of the average electron velocity due to the growth of the EM wave amplitude should be taken into account. For an illustration on how the gain in CFELs can be susceptible to variations of the electron beam velocity, we recall the bunch dynamics that provides the amplification. The forward electric field component of the transverse guided mode, pointing along the direction of the electron beam, can decelerate or accelerate the propagating electrons forming electron bunches on the scale of the EM wavelength. A net amplification can be observed when the velocity of bunches approximately fits with the phase velocity of the EM wave, and these bunches are positioned within the decelerating phases of the radiation wave. However, when the amplification of the EM wave increases by receiving energy from electrons, bunches must shift from the optimum driving phases. Therefore, this mechanism of out-of-phase synchronization decreases the amplification gradually.

Shiozawa ${ }^{33,34}$ studied the variation influence of the electron drift velocity on the gain of the CFELs using the coupled mode theory between the EM and the space charge waves. Also, the effect of phase velocity fluctuations of the radiation wave due to waveguide imperfections was investigated by de Fuente et al. ${ }^{35}$

In this paper, we present an analytical model to describe the amplification gain in CFELs with a planar waveguide evolving from the small-signal regime to saturation. We start with the Maxwell's wave equation to describe the amplification of the EM wave by the exciting electron current density in a similar fashion with the analyses of the previous study 
shown in Ref. 22. In this analysis, we newly take into account the electron velocity attenuation on the assumption of large amplitudes of oscillating fields. Also, the electron relaxation effect on modulations of electron velocity and density is considered. In the small-signal regime, we show that the operation of the stimulated emission with the spatial variation can be classified into two regions. In the first region of so-called transition state, the growth rate of the EM wave increases as the electrons travel down the waveguide. In the second region of so-called steady state, the growth rate does not change for further increase in travel distance of electrons. At saturation regime, the consequence of the reduction of the electron velocity could be suppression in the growth rate of the power gain in the steady state as well as in the transition state. For the small-signal regime, we prove that our results in the transition region agree well with those predicted from other treatments of free-electron lasers.

This paper is composed as follows. In Sec. II, we present the basic geometry of the CFEL configuration and formulations for the EM wave amplification by applying Maxwell's wave equation. In Sec. III, the effects of the electrons relaxation and the velocity attenuation are introduced into the dynamics of the electron beam to obtain the radiation growth characteristics. The spatially averaged gain is obtained and comparisons between our results and those of other treatments are introduced in Sec. IV. In Sec. V, conclusions are given.

\section{CFEL MODELING}

Before discussing the specifics aspects of the problem, we review some basic elements of formulations that describe the excitation of the EM wave by the current source of the electron beam. The basic model of the CFEL as an amplifier is illustrated in Fig. 1. In Fig. 1, an electron beam is running with a velocity $v_{\mathrm{el}}$ above a surface of a dielectric slab waveguide. In the low-gain regime, a feedback mechanism, e.g., a Fabry-Perot resonator, is required to enable efficient operation. An EM wave propagates along the waveguide with a phase velocity $v_{\mathrm{em}}$ and penetrates partly into the vacuum region to cross the electron beam. The interaction between electrons and EM wave is induced when $v_{\mathrm{el}} \approx v_{\mathrm{em}}$. The spontaneous emission (or an inputted EM wave) can gain more energy from electrons to stimulate further Cerenkov radiation. In the case of the slab waveguide, only the transverse magnetic (TM) modes are excited because the existence of a longitudinal electric field is required to interact with the electron beam.

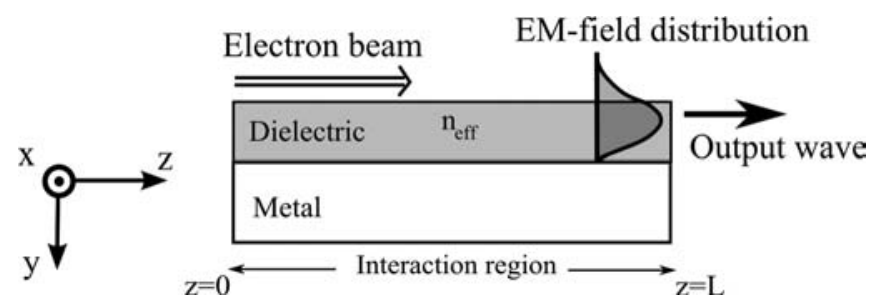

FIG. 1. Geometry of the electron beam-dielectric guide interaction in a CFEL as an amplifier. If two mirrors are added at both ends, the CFEL device will work as an oscillator.
The propagation constant $\beta$ of the EM wave propagating in the same direction of the electron beam is defined as

$$
\beta=\sqrt{\mu_{0} \varepsilon_{0}} \omega n_{\mathrm{eff}}=\frac{2 \pi n_{\mathrm{eff}}}{\lambda},
$$

where, $\varepsilon_{0}, \mu_{0}$, and $\lambda$ are the dielectric constant, the permeability, and the EM wavelength in free space, respectively. $n_{\text {eff }}$ is the effective refractive index whose values are obtained from the dispersion relation of the waveguide yielded by imposing the continuity of the fields at interfaces in Maxwell's equations for the TM polarization. Values of $n_{\text {eff }}$ are influenced by the dielectric film geometry and the refractive index of the film. Thus, the condition of synchronism at which an electron feels a nearly constant electric field can be written as

$$
v_{\mathrm{el}}=\frac{c}{n_{\mathrm{eff}}},
$$

where $c$ is velocity of the light in free space.

We assume that the evolution of the electric field $\boldsymbol{E}$ of the EM wave can be described by the classical wave equation, $^{36}$

$$
\nabla^{2} \boldsymbol{E}-\mu_{0} \varepsilon_{i} \frac{\partial^{2} \boldsymbol{E}}{\partial t^{2}}=\mu_{0} \frac{\partial \boldsymbol{J}_{z}}{\partial t}+\frac{1}{\varepsilon_{0}} \nabla \rho,
$$

where $\varepsilon_{i}$ is the dielectric constant in the $i$ th layer and $\rho$ and $\boldsymbol{J}_{z}$ are the charge and the current densities of the electron beam, respectively. The right-hand side of Eq. (3) is the source terms of the excitation of the forward propagating wave. Since we are basically concerned with the gain of the CFEL device, we neglected in Eq. (3), the contribution of the charge density in the waveguide that represents the loss mechanism "or the conductivity" in the waveguide material.

The electric field of the EM wave $\boldsymbol{E}$ can be expressed in the form

$$
\boldsymbol{E}=F(z) \boldsymbol{T}(x, y) e^{j(\omega t-\beta z)}+c . c .,
$$

where $F(z)$ is the field amplitude of the propagating wave whose spatial variation is much smoother than the phase variation of $\exp (-j \beta z) . \boldsymbol{T}(x, y)$ is the transverse electric field distribution which is the solution of the homogeneous equation

$$
\left[\nabla^{2}+\mu_{0} \varepsilon_{i} \omega^{2}\right] \boldsymbol{T}(x, y) e^{-j \beta z}=0,
$$

and satisfies the normalization condition in the form of

$$
\begin{aligned}
\int_{-\infty}^{\infty} & \int_{-\infty}^{\infty}|\mathbf{T}(x, y)|^{2} d x d y \\
& =\int_{-\infty}^{\infty} \int_{-\infty}^{\infty}\left(\left|T_{y}(x, y)\right|^{2}+\left|T_{z}(x, y)\right|^{2}\right) d x d y=1 .
\end{aligned}
$$

By substituting Eq. (4) into Eq. (3), by using relations of Eqs. (5) and (6) after multiplying both sides of Eq. (3) by $\boldsymbol{T}^{*}(x, y) \exp [j(\beta z-\omega t)]$, and by neglecting the second derivative of $F(z)$, we obtain the variation of the field amplitude considering the spatial and time averages in the form of 


$$
\begin{aligned}
\frac{\partial F(z)}{\partial z}= & j \frac{1}{2 \beta} \frac{1}{\Delta t \Delta z} \int_{t-\Delta t}^{t} \int_{0}^{\Delta z} \int_{-\infty}^{\infty} \int_{-\infty}^{\infty}\left[\mu_{0} \frac{\partial J_{z}}{\partial t}+\frac{1}{\varepsilon_{0}} \nabla \rho\right] \\
& \times \boldsymbol{T}^{*}(x, y) e^{j \beta z-j \omega t} d x d y d z d t
\end{aligned}
$$

where $\Delta z$ is a spatial interval to take the spatial average and is sufficiently longer than the EM wavelength but the amplitude of the EM field is assumed to be constant over the interval $\Delta z . \Delta t$ is a time interval used to take the time average and should be sufficiently greater than several EM oscillation periods. Since the axial bunching is the key physical processes for the radiation generation, we consider only the axial motion of the electrons. So that, given the beam is directed along $\mathrm{z}$-direction, the electron beam interacts with the longitudinal electric field component $E_{z}$ and the transverse field distribution $T_{z}(x, y)$ is only taken into account on the righthand side of Eq. (7). From Eq. (7), the variation of the field amplitude of the radiation wave can be written as

$$
\frac{\partial F(z)}{\partial z}=g F(z)
$$

and

$$
\begin{aligned}
g= & -\frac{1}{2 \beta} \operatorname{Im}\left\{\frac{1}{F(z)} \frac{1}{\Delta t \Delta z} \int_{t-\Delta t}^{t} \int_{0}^{\Delta z} \int_{-\infty}^{\infty} \int_{-\infty}^{\infty}\right. \\
& \left.\times\left[\mu_{0} \frac{\partial J_{z}}{\partial t}+\frac{1}{\varepsilon_{0}} \frac{\partial \rho}{\partial z}\right] T_{z}^{*}(x, y) e^{j \beta z-j \omega t} d x d y d z\right\},
\end{aligned}
$$

where $g$ is the spatial growth rate or the gain coefficient of the guided wave.

From the continuity equation of charged particles

$$
\nabla \cdot J_{z}=-\frac{\partial \rho}{\partial t}
$$

we can get the relation of $\partial \rho / \partial z=\left(\partial J_{z} / \partial t\right)(\partial t / \partial z)^{2}$ $=\left(\partial J_{z} / \partial t\right) / v_{\mathrm{el}}^{2}{ }^{18}$ Since the electron velocity is almost same as the phase velocity $v_{\mathrm{em}}=c / n_{\mathrm{eff}}$ as depicted in Eq. (2), the term in the bracket [ ] of Eq. (9) can be approximated to be $\left(1+n_{\text {eff }}^{2}\right) \mu_{0} \partial J_{z} / \partial t$. Then, Eq. (9) becomes

$$
\begin{aligned}
g= & -\frac{\left(1+n_{\mathrm{eff}}^{2}\right) \mu_{0}}{2 \beta} \operatorname{Im}\left\{\frac{1}{F(z)} \frac{1}{\Delta t \Delta z} \int_{t-\Delta t}^{t} \int_{0}^{\Delta z} \int_{-\infty}^{\infty}\right. \\
& \left.\times \int_{-\infty}^{\infty} \frac{\partial J_{z}}{\partial t} T_{z}^{*}(x, y) e^{j \beta z-j \omega t} d x d y d z\right\} .
\end{aligned}
$$

In our analyses, we subsume the amplification of the EM wave (or attenuation) into the positive (or negative) values of the gain coefficient.

\section{STIMULATED EMISSION AND AMPLIFICATION}

\section{A. Dynamic motion of electrons and gain coefficients}

The effect of electron-electron collisions in the electron beam is an important factor that influences the dynamics of electrons. The electrons which are assumed to be uniformly distributed in the electron beam will be subjected to symmetric Coulomb repulsive forces from neighboring electrons. After modulations induced from the longitudinal electric field component of the EM wave, the separating distances between electrons must be unequal. Then, each electron will be subjected to different Coulomb's forces from the adjacent electrons. These forces attempt to steer the electrons to its initial positions and relax the electron velocity to its average value. This process is termed as electron relaxation.

Variation of the electron velocity $v$ can be described by non-relativistic equation of motion for the electron, ${ }^{12,22,24}$

$$
\frac{d v}{d t}=\frac{\partial v}{\partial t}+v \frac{\partial v}{\partial z}=-\frac{e}{m_{o}}\left[E_{z 0} e^{g z+j(\omega t-\beta z)}+c . c .\right]-\frac{v-\bar{v}}{\tau},
$$

where $-e$ and $m_{0}$ are electron charge and electron mass, respectively. In Eq. (12), by the help of Eq. (8), the electric field component $E_{z}$ in Eq. (4) is rewritten as $E_{z 0} e^{g z+j(\omega t-\beta z)}$ $+c . c$ assuming $E_{z 0}=F(0) T_{z}(x, y)$. The first term on the right-hand side of Eq. (12) represents that the modulation of the electron velocity is effectively influenced by $E_{z}$ which exerts the Lorentz force on the electron charge, while the second term corresponds to the damping phenomenon of the electron velocity to a timely averaged velocity $\bar{v}$ characterized by the relaxation time $\tau$.

Variation of electrons density $N$ is introduced by help of the continuity equation given by Eq. (10) such as ${ }^{12,22,24}$

$$
\frac{\partial N}{\partial t}=-\frac{\partial}{\partial z}(N v)-\frac{N-\bar{N}}{\tau},
$$

where $\bar{N}$ is a timely averaged electron density. The first term on the right-hand side of Eq. (13) expresses the physical fact that the total number of electrons must be conserved, whereas any decrease in the electron number inside an arbitrary volume with time must correspond to a flow of electrons going out through the surface of this volume. The relaxation effect introduced by the second term on the right-hand side of Eq. (13) will attempt to relax the electron density $N$ at time $t$ to the initial value $\bar{N}$ due to the coulomb scatterings among electrons being unequally spaced. Since the velocity modulation causes the density bunching in the electron beam along the interaction region, we assume that the relaxation time $\tau$ is identical for the velocity and the density modulations in an approximate manner.

In our treatment, a basic task is to obtain the timely rate of the stimulating current density shown in Eq. (11), which can be written as

$$
\frac{\partial J_{\mathrm{z}}}{\partial t}=-e \frac{\partial(N v)}{\partial t}
$$

For this purpose, we propose that the modulated velocity can be expanded as the first order approximation in a form of

$$
v=\bar{v}(z)+\left[u(z) e^{j \omega t-(j \omega+1 / \tau) z / \bar{v}}+c . c .\right] .
$$

Similarly, the modulated density will be given as

$$
N=\bar{N}(z)+\left[n(z) e^{j \omega t-(j \omega+1 / \tau) z / \bar{v}}+c . c .\right],
$$

where $u(z)$ and $n(z)$ are spatially dependent coefficients of velocity and density modulations, respectively. These coefficients are assumed to be a slowly varying in comparing with the spatial phase variation of the EM wave. In Eqs. (15) and 
(16), we consider that the average velocity $\bar{v}(z)$ and density $\bar{N}(z)$ of the electron beam are varied as the EM wave grows at the expense of the kinetic energy of the electron beam.

Now, we will embark on getting the modulation indices of the electron velocity and density $u(z)$ and $n(z)$, respectively. By substituting Eq. (15) into Eq. (12) and by comparing the fast and slowly varying components on both sides, we can get a couple of equations as

$$
\frac{\partial \bar{v}(z) u(z)}{\partial z}=-\frac{e}{m_{o}} E_{z 0} e^{(-j \Omega+g \bar{v}+1 / \tau) z / \bar{v}},
$$

and

with

$$
\bar{v}(z) \frac{\partial \bar{v}(z)}{\partial z}=-\frac{\partial}{\partial z}\left[|u(z)|^{2} e^{\frac{-2 z}{\bar{v} \tau}}\right]
$$

$$
\Omega(z)=\beta \bar{v}(z)-\omega,
$$

where $\Omega(z)$ is the wave frequency as seen by the electron for which the exact synchronism is exhibited when $\Omega(z)=0$.

By the help of Eq. (17a), after performing the integration from $z=0$ to $z=z$ and by taking into account the initial condition of $u(0)=0$, we can get $u(z)$ as

$$
u(z)=-\frac{e}{m_{o}} \frac{E_{z 0}}{[-j \Omega+(g \bar{v}+1 / \tau)]}\left[e^{(-j \Omega+g \bar{v}+1 / \tau) z / \bar{v}}-1\right] .
$$

From Eq. (17b), the variation in the average velocity of the electron beam is given by

$$
\begin{aligned}
\bar{v}^{2}(z)= & \bar{v}^{2}(0)-2\left(\frac{e}{m_{o}}\right)^{2} \frac{\left|E_{z}\right|^{2}}{\left[\Omega^{2}+(g \bar{v}+1 / \tau)^{2}\right]} \\
& \times\left[1-2 e^{-(g \bar{v}+1 / \tau) \frac{z}{\bar{v}}} \cos (\Omega z / \bar{v})+e^{-(g \bar{v}+1 / \tau) \frac{2 z}{\bar{v}}}\right] .
\end{aligned}
$$

According to Eq. (17b), the reduction in the electron velocity shown in Eq. (20) is proportional to the EM field intensity and is taken into account by counting contribution of $|u(z)|^{2}$. At saturation, the variation of the average electron velocity has a significant effect on the relative angular frequency $\Omega(z)$ causing the phenomenon of limited gain.

By substituting Eq. (16) into Eq. (13) and by separating the fast and slowly varying components, we can get from the continuity equation:

$$
\frac{\partial[\bar{v}(z) n(z)]}{\partial z}=1 / \bar{v}(j \omega+1 / \tau) \bar{N}(z) u(z)-\frac{\partial[\bar{N}(z) u(z)]}{\partial z},
$$

and

$$
\frac{\partial[\bar{N}(z) \bar{v}(z)]}{\partial z}=-\frac{\partial\left\{\left[n(z) u^{*}(z)+u(z) n^{*}(z)\right] e^{-2 z / \bar{v} \tau}\right\}}{\partial z} .
$$

On the right-hand side in Eq. (21a), by neglecting the second term in comparison with the first term, by putting $(j \omega+1 / \tau) \approx j \beta \bar{v}$ and by performing the spatial integration from $z=0$ to $z=z$, we obtain

$$
n(z)=-\frac{j \bar{N}(z) \beta e E_{z 0}}{m_{0}[-j \Omega+(g \bar{v}+1 / \tau)]}\left[\frac{e^{(-j \Omega+g \bar{v}+1 / \tau) z / \bar{v}}-1}{[-j \Omega+(g \bar{v}+1 / \tau)]}-z / \bar{v}\right] .
$$

To get Eq. (22), we also used the initial condition of $n(0)=0$.

Here, it would be helpful to get an expression for $\bar{N}(z) \bar{v}(z)$ by using Eq. (21b), such as

$$
\begin{aligned}
\bar{N}(z) \bar{v}(z)= & \bar{N}(0) \bar{v}(0)-j\left(\frac{e}{m_{0}}\right)^{2} \frac{\bar{N}(0) \beta\left|E_{z}\right|^{2}}{\left[\Omega^{2}+(g \bar{v}+1 / \tau)^{2}\right]}\left[\frac{2 j \Omega\left[1+e^{-2(g \bar{v}+1 / \tau) z / \bar{v}}\right]-4 j \Omega e^{-(g \bar{v}+1 / \tau) z / \bar{v}} \cos (\Omega z / \bar{v})}{\left[\Omega^{2}+(g \bar{v}+1 / \tau)^{2}\right]}\right. \\
& \left.-2 j \frac{z}{\bar{v}} e^{-(g \bar{v}+1 / \tau) z / \bar{v}} \sin (\Omega z / \bar{v})\right]
\end{aligned}
$$

The first term on the right-hand side in Eq. (23) represents the unperturbed average current density corresponding to the case of small-signal approximation, while the second term corresponds to the variation of the average current density due to a significant increase in the EM field amplitude in the saturation regime.

The time derivative of the current density $\partial J_{z} / \partial t$ shown in Eq. (14) can be obtained by the help of Eqs. (15), (16), (19), and (22) such as

$$
\begin{aligned}
\frac{\partial J_{z}}{\partial t}= & \frac{j \omega e^{2} \bar{N}(z) E_{z}}{m_{o}[-j \Omega+g \bar{v}+1 / \tau]} \times\left\{1-e^{[j \Omega-(g \bar{v}+1 / \tau)] z / \bar{v}}\right\} e^{j(\omega t-\beta z)} \\
& -\frac{\omega e^{2} \bar{N}(z) \bar{v}(z) \beta E_{z}}{m_{o}[-j \Omega+g \bar{v}+1 / \tau]} \times\left\{\frac{1-e^{[j \Omega-(g \bar{v}+1 / \tau)] z / \bar{v}}}{[-j \Omega+g \bar{v}+1 / \tau]}\right. \\
& \left.-e^{[j \Omega-(g \bar{v}+1 / \tau)] z / \bar{v}} z / \bar{v}\right\} e^{j(\omega t-\beta z)}+c . c .
\end{aligned}
$$

Since $(-j \Omega+g \bar{v}+1 / \tau) \ll \beta \bar{v}$, the first term in Eq. (24) can be neglected, and we get more compact form for the time variation of the current density as

$$
\begin{aligned}
\frac{\partial J_{z}}{\partial t} \approx & -\frac{e^{2} \omega \bar{N}(z) \bar{v}(z) \beta E_{z}}{m_{o}\left(1 / \tau^{2}\right)[j \Omega \tau-(g \bar{v} \tau+1)]^{2}} \\
& \times\{1-[1-(j \Omega \tau-g \bar{v} \tau-1)(z / \bar{v} \tau)] \\
& \left.\times e^{[j \Omega \tau-(g \bar{v} \tau+1)] \overline{\bar{v}}}\right\} e^{j(\omega t-\beta z)} .
\end{aligned}
$$

As a result of variation in the average velocity $\bar{v}(z)$, the relative angular frequency $\Omega(z)$ defined in Eq. (18) as $\Omega(z)=\beta \bar{v}(z)-\omega$ is also varied. Since values of $\Omega(z)$ are very close to 0 , the relative variation of $|\delta \Omega(v) / \Omega(0)|$ becomes very large. The variation of $\Omega(z)$ mostly affects on the variation of the gain coefficient as shown in later. In Eq. 
(25), the term $\bar{v}(z) \tau$ gives the interaction length during the relaxation time $\tau$ and the relative variation of $|\delta \bar{v}(z) / \bar{v}(0)|$ is much smaller than that of $|\delta \Omega(v) / \Omega(0)|$. Then, the variation of $\bar{v}(z) \tau$ is insignificant in comparison with the variation of $\Omega(z)$. In following calculations, we count the z-dependence of the terms $\bar{N}(z) \bar{v}(z), \Omega(z)$ and $g(z)$, while we put $\bar{v}(z) \tau \approx \bar{v}(0) \tau$ as an approximation for simple treatments.
By substituting the time variation of the current density given by Eq. (25) into Eq. (11) and by recalling the expression of $\bar{N}(z) \bar{v}(z)$ given by Eq. (23), we can express the gain coefficient defined in Eq. (11) by the following equation:

$$
g=g_{1}+g_{2},
$$

where

$$
g_{1}=\frac{\left(1+n_{\mathrm{eff}}^{2}\right) e^{2} \mu_{0} \omega \bar{N}(0) \bar{v}(0)}{m_{o}} \xi_{1} \operatorname{Im}\left\{\tau^{2} \times \frac{\left\{1-\left[1-\left(j \Omega \tau-g l_{\mathrm{sc}}-1\right)\left(z / l_{\mathrm{sc}}\right)\right] e^{\left[j \Omega \tau-\left(g l_{\mathrm{sc}}+1\right)\right]}\right\}}{\left[j \Omega \tau-\left(g l_{\mathrm{sc}}+1\right)\right]^{2}}\right\}
$$

with

$$
\xi_{1}=\int_{-w}^{0} \int_{-w / 2}^{w / 2}\left|T_{z}(x, y)\right|^{2} d x d y
$$

and

$$
\begin{aligned}
g_{2}= & -\left(1+n_{\mathrm{eff}}^{2}\right)\left(\frac{e^{4}}{m_{0}^{3}}\right) \mu_{0} \omega \beta \bar{N}(0)|F(z)|^{2} \xi_{2} \\
& \times \operatorname{Re}\left\{\begin{array}{l}
\tau^{5} \times \frac{\left\{1-\left[1-\left(j \Omega \tau-g l_{\mathrm{sc}}-1\right)\left(z / l_{\mathrm{sc}}\right)\right] e^{\left[j \Omega \tau-\left(g l_{\mathrm{sc}}+1\right)\right]}\right\}}{\left[j \Omega \tau-\left(g l_{\mathrm{sc}}+1\right)\right]^{2}\left[(\Omega \tau)^{2}+\left(g l_{\mathrm{sc}}+1\right)^{2}\right]} \\
\times\left[\frac{2 j \Omega \tau\left[1+e^{\left.-2\left(g l_{\mathrm{sc}}+1\right) z / l_{\mathrm{sc}}\right]}-4 j \Omega \tau e^{-\left(g l_{\mathrm{sc}}+1\right) z / \mathrm{sc}_{\mathrm{sc}} \cos \left(\Omega \tau z / l_{\mathrm{sc}}\right)}\right.}{\left[(\Omega \tau)^{2}+\left(g l_{\mathrm{sc}}+1\right)^{2}\right]}-2 j \sin \left(\Omega \tau z / l_{\mathrm{sc}}\right) e^{-\left(g l_{\mathrm{sc}}+1\right) z / l_{\mathrm{sc}}} \frac{z}{l_{\mathrm{sc}}}\right]
\end{array}\right\},
\end{aligned}
$$

with

$$
\xi_{2}=\int_{-w}^{0} \int_{-w / 2}^{w / 2}\left|T_{z}(x, y)\right|^{4} d x d y
$$

Here, $l_{\mathrm{sc}}=\bar{v}(0) \tau$ is the distance that an electron travels during the relaxation time $\tau$. $\xi_{1}$ and $\xi_{2}$ are coupling coefficients between the electron beam and the evanescent part of the EM wave. By introducing the coefficients $\xi_{1}$ and $\xi_{2}$, the variation of the electric field over the cross-sectional area of the electron beam $w \times w$ in the transverse $\mathrm{x}$ - and $\mathrm{y}$-directions is taken into account.

The gain coefficient $g$ described by Eq. (26) is divided into two different sub-coefficients. The first one is $g_{1}$ which has a similar form to that given in Ref. 22 for the smallsignal approximation, while the second term $g_{2}$ is newly obtained by counting the term of $\left[n(z) u^{*}(z)+u(z) n^{*}(z)\right]$ in Eq. (21b). It is worth to note that the saturation effect is even included in the linear gain coefficient $g_{1}$, whereas the relative electron phase $\Omega(z)$ varies spatially due to the variation of the average velocity given by Eq. (20).

For the small-signal analysis, by considering $g l_{\mathrm{sc}} \ll 1$ and $\Omega(z) \approx \Omega(0)$, whereas $|u(z)|^{2} \approx 0$, the gain coefficient $g_{1}$ will be given by

$$
\begin{aligned}
g_{1}= & \frac{\left(1+n_{\mathrm{eff}}^{2}\right) e^{2} \mu_{0} \omega \bar{N}(0) \bar{v}(0)}{m_{o}} \xi_{1} \\
& \times \operatorname{Im}\left\{\tau^{2} \frac{\left\{1-\left[1-(j \Omega(0) \tau-1)\left(z / l_{\mathrm{sc}}\right)\right] e^{[j \Omega(0) \tau-1] \frac{z}{\mathrm{sc}}}\right\}}{[j \Omega(0) \tau-1]^{2}}\right\} .
\end{aligned}
$$

In the small-signal gain, since we can neglect the contributions of the term $\left[n(z) u^{*}(z)+u(z) n^{*}(z)\right]$, thus we can assume $g_{2} \approx 0$.

In a previous quantum mechanical analysis of the CEFL, ${ }^{21}$ the authors proposed that the electron can be represented as a plane wave with a finite length. In that quantum mechanical model, the electron relaxation effect is represented as a damping phenomenon on the time vibration of the electron wave. The electron relaxation time was estimated to be in the order of $10^{-10}-10^{-9} \mathrm{~s}$. The electron relaxation time in the quantum mechanical model should be smaller than that defined in this paper for the electron considered as a point particle. In Sec. II B, to get an appreciation for the level of gain predicted in our calculations, we assume that the relaxation time takes the same value as that obtained in the quantum mechanical analysis for a preliminary approximation.

\section{B. Numerical examples and discussions}

Now, we start to determine the gain coefficient given by Eq. (29) under the small-signal approximation. Numerical examples, depicted in Fig. 2, is used to explore the variation of the gain coefficient $g_{1}$ with the normalized distance $z / l_{\text {sc }}$. In this example, we assumed that $\lambda=1 \mathrm{~cm}, n_{\text {eff }}=3.0$, $\xi_{1}=0.1, \quad \tau=10^{-9} \mathrm{~s}, \quad$ and $\quad \bar{J}=10^{4} \mathrm{~A} / \mathrm{m}^{2}, \quad$ where $\bar{J}=e \bar{N}(0) \bar{v}(0)$ is the dc component of the electron beam current density. In these examples of the small-signal approximation, the average electron velocity is supposed to be constant along the interaction length. In Fig. 2, the gain coefficient increases almost linearly with the travel distance of electrons $z$ up to several times of $l_{\text {sc }}$ and takes constant 


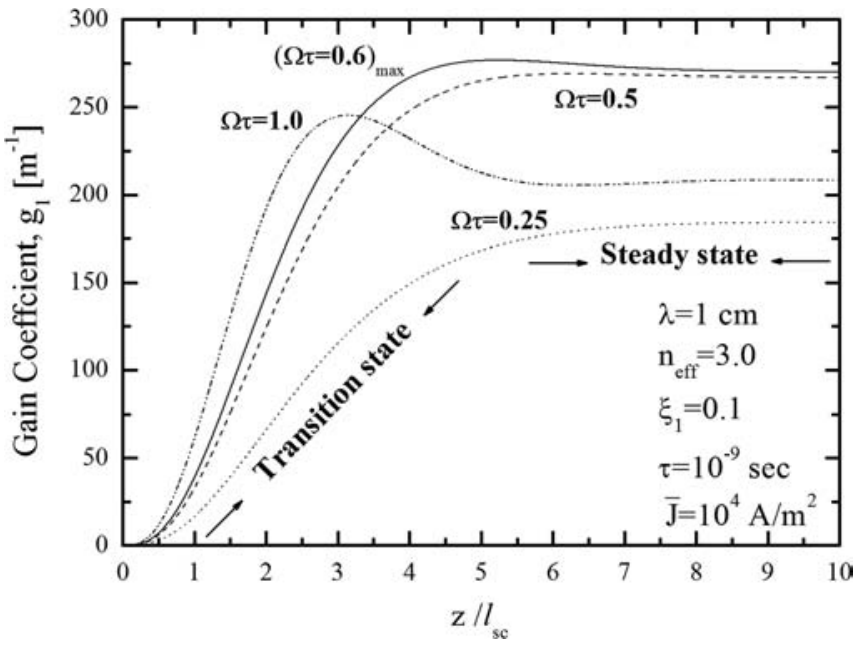

FIG. 2. Variations of the gain coefficient with the normalized distance $z / l_{\mathrm{sc}}$ for different values of $\Omega \tau$ in the small-signal approximation. The interaction regions induced by the stimulated emission can be divided into transition and steady states. In the transition state, the gain coefficient increases almost linearly with the spatial variation. In the steady state, when the passing distance $z$ reaches several times of the distance $l_{\mathrm{sc}}$, the gain coefficient saturates at certain values.

values for further distance increase. The behavior of the interaction induced by the stimulated emission can be classified into two ranges. We call the former range "the transition state" and the latter range "steady state". As seen in Fig. 2, the range of the transition sate or the steady state can be determined by examining whether the interaction time $t=z / \bar{v}$ is approximately shorter or longer than twice of the relaxation time $2 \tau$, respectively. In the transition state, as the electron travels down the waveguide, the gain coefficient $g_{1}$ increases with the enhancement of the electron beam modulations. In the same time, the relaxation effect works to reduce the modulations rate. In the steady state region when the wave amplitude becomes sufficiently high with strong bunching, any further increase in the modulation will be eliminated by the relaxation effect, and the gain coefficient becomes almost constant. We also can observe that the gain coefficient have maximum values at $\Omega(0) \tau=0.6$ in the steady state.

In the saturation regime, let us discuss the spatial variation of the gain coefficient $g$ described by Eqs. (26)-(28). In Fig. 3(a), we report variations of the gain coefficients $g_{1}$ and $g_{2}$ along the normalized distance $z / l_{\mathrm{sc}}$ when $\Omega(0) \tau=1$. The velocity-related variation of the effective phase $\Omega(z)$ is shown in Fig. 3(b). In this example, we assume that the existing electric field of $\left|E_{z 0}\right|=10^{5} \mathrm{~V} / \mathrm{m}$ and the input power of $P(0)=10^{3} \mathrm{~W}$ with the coupling coefficient $\xi_{2}=0.01 \mathrm{~m}^{-2}$. Other remaining parameters takes the same values used to draw Fig. 2. The variation of the field intensity $|F(z)|^{2}$ is traced along the axial distance by using the relation of $|F(z)|^{2} \approx\left(1 / 2 n_{\text {eff }}\right) \sqrt{\mu_{0} / \varepsilon_{0}} P(z) \cdot{ }^{21,22}$ In Fig. 3(a), the gain coefficient $g_{1}$ increases with increasing $z / l_{\mathrm{sc}}$ until the gain coefficient reaches to a maximum value. After that, as the power growth of the EM wave becomes sufficiently high at the expense of the kinetic energy of electrons, out-of-phase synchronization given by $\Omega(z)$ between the electrons bunches and the EM wave leads to significant degradation in (a)

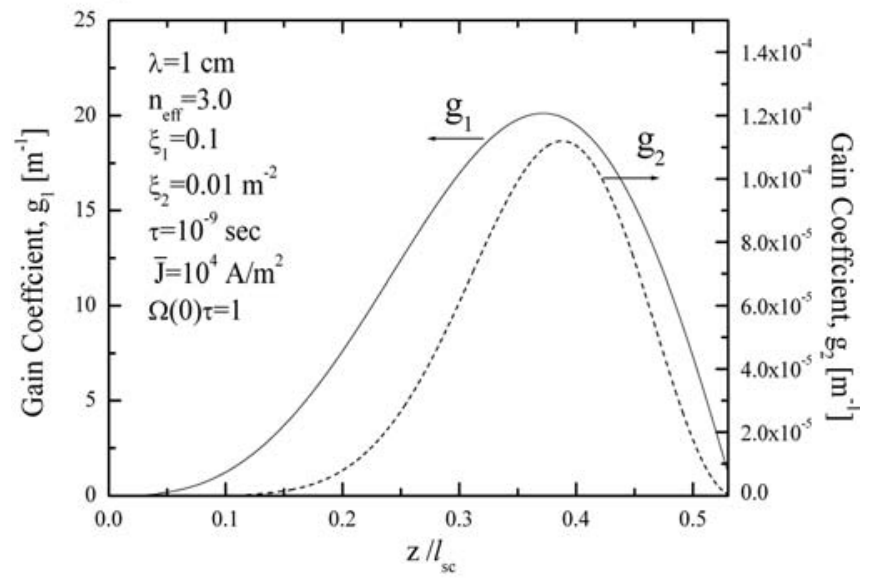

(b)

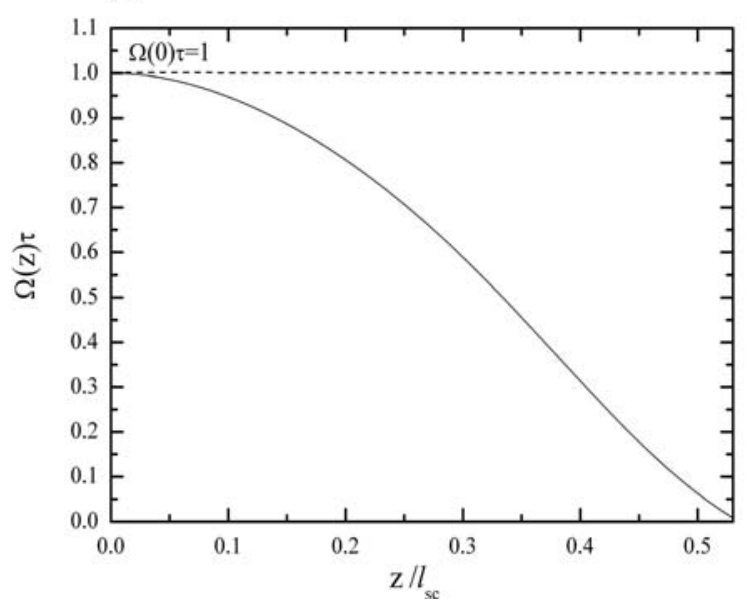

FIG. 3. Numerical examples of the saturated gains and the relative angular frequency with the normalized distance $z / l_{\mathrm{sc}}$. (a) Variations of the gain coefficients $g_{1}$ and $g_{2}$. A high input power of $P(0)=10^{3} \mathrm{~W}$ is supposed to clarify the saturation effect. $g_{1}$ increases along the electron beam and reaches a maximum value and after that it will gradually decrease due to the shifting of the averaged velocity of the electron beam from the synchronism condition. The gain coefficient $g_{2}$ is proportional to the power of the EM wave; thus, it have the same behavior as for the gain coefficient $g_{1}$. (b) The attenuation of the wave frequency $\Omega(z)$ as seen by traversing electrons vs. the normalized distance $z / l_{\mathrm{sc}}$.

the gain coefficient. Moreover, the gain coefficient $g$ in the denominator of Eqs. (27) and (28) also causes selfsuppression in the gain coefficient. Since the gain coefficient $g_{2}$ is proportional to the propagating power $P(z), g_{2}$ will follow the same behavior of $g_{1}$.

In Fig. 4, we illustrate the variations of the gain coefficient $g_{1}$ for different values of $\Omega(0) \tau$. The gain coefficient no longer shows maximum values at $\Omega \tau=0.6$ as occurred in the case of the small-signal shown in Fig. 2, because the gain cannot reach to the steady state under the reduction influence of $\Omega(z)$.

Next, in Fig. 5, we compare values of the gain coefficient $g_{1}$ in the saturation regime described by Eq. (27) and that in the small-signal analysis given by Eq. (29). In the case of the small-signal analysis, the gain coefficient increases rapidly with the interaction length, because the operation is in the transition state shown in Fig. 2. Under saturation, by assuming that $g l_{\text {sc }} \ll 1$, we also draw the gain 


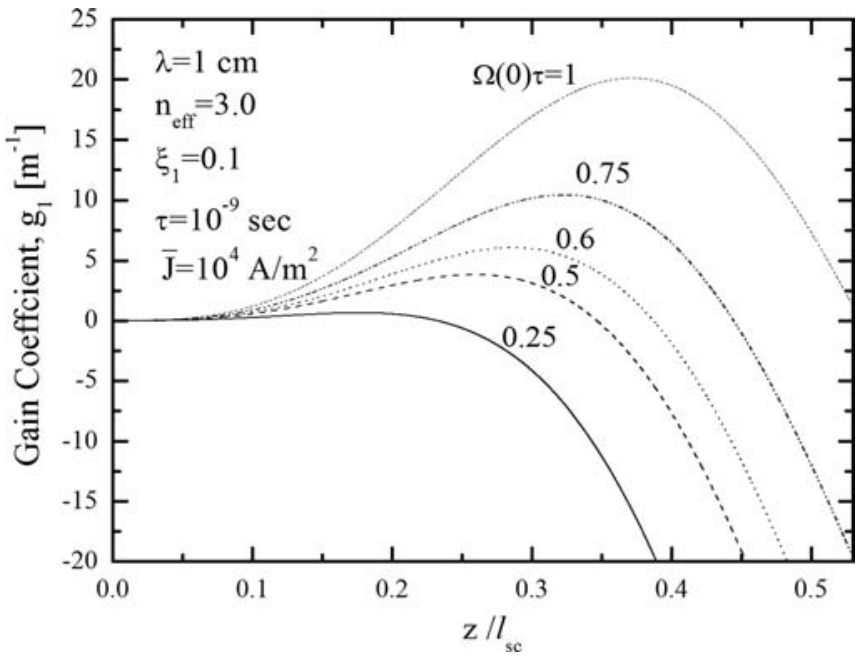

FIG. 4. Variations of the gain coefficient $g_{1}$ with $z / l_{\mathrm{sc}}$ for different values of $\Omega(0) \tau$ in the saturation regime. Due to the attenuation of the averaged velocity of electrons $\bar{v}(z)$, the gain cannot reach to the steady state region observed in the small-signal analysis and its maximum values occur at larger distances for larger values of $\Omega(0) \tau$.

coefficient $g_{1}$ as depicted by the dashed line. In the case of saturation regime, it can be seen in Fig. 5 that the initial values of the gain $g_{1}$ given by Eq. (27) at the beginning of interaction is almost similar with or without neglecting the condition of $g l_{\mathrm{sc}} \ll 1$, while a slight difference is observed after the gain reaches to the maximum value. Hence, we can confirm that the effect of the variation of $\Omega(z)$ is more pronounced than that of the term $g l_{\mathrm{sc}}$ appeared in the denominator of Eq. (27).

The given formulations of the gain coefficients in Eqs. (27) and (28) are used to get an expression for the spatial variation of the power gained by the EM wave $P(z)$, as

$$
P(z)=P(0) \exp \left[\int_{0}^{z} 2\left(g_{1}+g_{2}\right) d z\right] .
$$

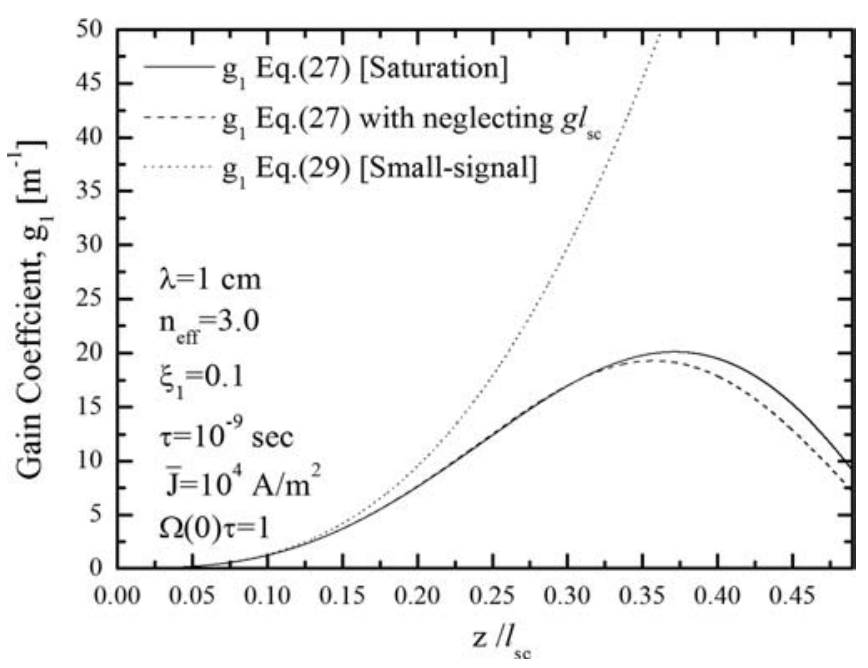

FIG. 5. Comparison between the gain coefficient $g_{1}$ in the small-signal gain and saturation limits. The small-signal case is represented by dotted line. At saturation, numerical solutions to the gain coefficient given by Eq. (27) is shown by solid line and by dashed line when the term of $g l_{\mathrm{sc}}$ is neglected in the denominator of Eq. (27).
The factor 2 in the above equation corresponds to the fact that the power of the EM wave $P(z)$ is proportional to square of the amplitude given by Eq. (8). Numerical examples of the power ratio $P(z) / P(0)$ versus the normalized distance $z / l_{\mathrm{sc}}$ are shown in Fig. 6 . In these examples, we assume the same parameters used to draw Fig. 3. In Fig. 6, we numerically calculate the power ratio $P(z) / P(0)$ given by Eq. (30) by the help of Eqs. (27)-(29) in the saturation and smallsignal approaches, respectively. For saturation treatment, we also draw the ratio power variations when the coefficient $\Omega$ is considered as constant, i.e., $\Omega(z)=\Omega(0)$, this case is depicted by the solid line. As shown by the dotted line in Fig. 6, the behavior of the gain coefficient in the small-signal approximation predicts an infinite exponential growth in the radiation power with the propagation distance as expected from the relation $P(z)=P(0) \exp \left(2 g_{1} z\right)$. The operation of saturation depicted by the dashed line, the power ratio increases linearly and tends to decrease after reaching a maximum value when the suppression of growth rate becomes sufficiently large. If the EM field intensity is not sufficiently high keeping the average electron velocity $\bar{v}(z)$ almost constant, the power ratio $P(z) / P(0)$ can only vary linearly with the traveling distance without declination, as shown by the solid line in Fig. 6.

It would be useful to point out that the evolutions of the power and the gain with the interaction length shown in the numerical examples of this section are well-matched with those expected from the analytical results in Ref. 28 in which the effect of beam thermal spread is taken into account. However, the initial energy spread should be one cause of the electron relaxation mechanism in the present paper.

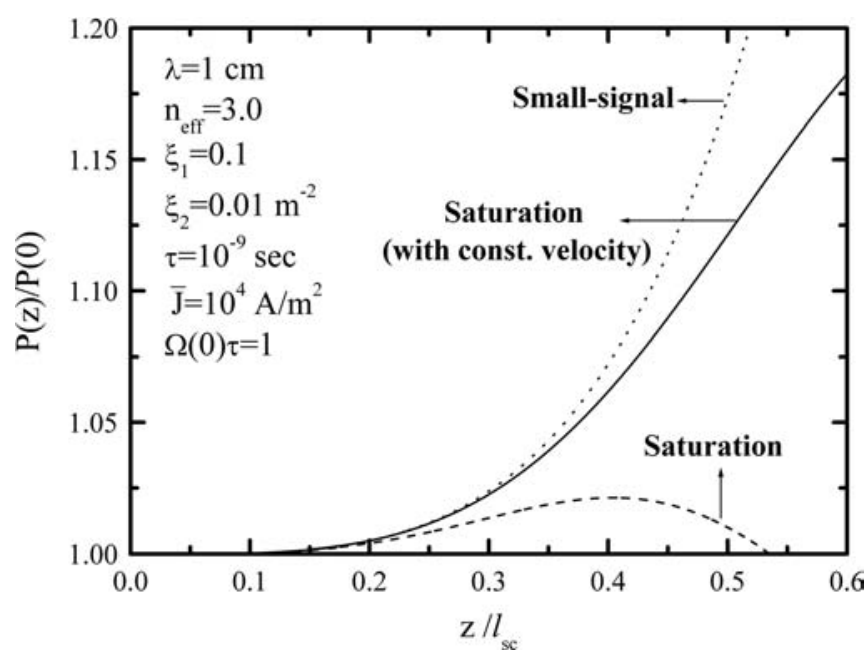

FIG. 6. Spatial variation of the power amplification $P(z) / P(0)$. The smallsignal gain is depicted by the dotted line, whereas the power amplification increases in the form of the exponential function. In the case of the saturation regime, the power amplification increases almost linearly with the normalized interaction length $z / l_{\mathrm{sc}}$ and decreases after reaching a maximum value as shown by the dashed line. In the saturation regime, if the averaged electron velocity is not significantly reduced, the amplification power would simultaneously vary linearly with the interaction length as shown by the solid line. 


\section{SPATIALLY AVERAGED GAIN COEFFICIENT}

Here, we take the spatial average of the gain coefficient over the interaction length, whereas the interaction length is considered to be the waveguide length $L$. By neglecting the small contribution of $g_{2}$, the spatially averaged gain can be written as

$$
\overline{g(\Omega, L)} \approx \frac{1}{L} \int_{0}^{L} g_{1}(z) d z=\frac{\left(1+n_{\mathrm{eff}}^{2}\right) \mu_{0} e \bar{J} \omega \xi_{1}}{m_{0}}(L / \bar{v})^{2} \times f(\Omega, L),
$$

where $f(\Omega, L)$ is the dispersion function defined as

$$
f(\Omega, L)=\operatorname{Im}\left\{\frac{2\left[1-e^{\left(j \Omega-g_{1} \bar{v}-1 / \tau\right) L / \bar{v}}\right]+\left\{\left(j \Omega-g_{1} \bar{v}-1 / \tau\right)(L / \bar{v})\left[1+e^{\left(j \Omega-g_{1} \bar{v}-1 / \tau\right) L / \bar{v}}\right]\right\}}{\left(j \Omega-g_{1} \bar{v}-1 / \tau\right)^{3}(L / \bar{v})^{3}}\right\} .
$$

We suppose that the relaxation effect caused by Coulomb repulsive forces among electrons can be neglected. In other words, the relaxation time becomes infinitely long in Eq. (32). When the relaxation effect is neglected and taking the condition of $g_{1} L \ll 1$ for the small-signal approximation, the dispersion function reduces to

$$
\begin{aligned}
\left.f(\Omega \Delta T)\right|_{\Delta T \ll \tau} & =\operatorname{Im}\left[\frac{2\left\{1-e^{j \Omega \Delta T}\right\}+j \Omega \Delta T\left\{1+e^{j \Omega \Delta T}\right\}}{(j \Omega)^{3}(\Delta T)^{3}}\right] \\
& =\frac{1}{\Omega \Delta T}\left[\frac{\sin ^{2}(\Omega \Delta T)}{(\Omega \Delta T / 2)^{2}}-\frac{\sin (\Omega \Delta T)}{\Omega \Delta T}\right]
\end{aligned}
$$

where $\Delta T=L / \bar{v}$ is the interaction time counted as the time interval for passing the waveguide. The dispersion function shown in Eq. (33) is well-known dispersion function in the theory of free-electron lasers. The dispersion function $\left.f(\Omega \Delta T)\right|_{\Delta T \ll \tau}$ is an antisymmetric function for $\Omega \Delta T$, and its absolute value takes the maximum value at 0.135 at $\Omega \Delta T= \pm 2.6$.

In the previously performed analyses, ${ }^{33,37}$ the authors assumed the linear distribution of the electron beam on the assumption of small amplitudes of oscillating fields. The basic feature of these analyses is to calculate the averaged variation of the energy carried by the electron after traveling a certain distance basing on the single electron model. In Refs. 33 and 37, by using the symbols of the current paper, the power exchange with the electron beam has been derived as

$$
\Delta P=\frac{e \omega I}{2 m_{0}}(\Delta T)^{3} \times\left.\left|E_{z}\right|^{2} f(\Omega, \Delta T)\right|_{\Delta T \ll \tau} .
$$

By replacing the electron beam current in term of current density $I=\iint_{w \times w} \bar{J} d x d y$ and by using the relation of $P(z)$ $\approx 2 \sqrt{\varepsilon_{0} / \mu_{0}} n_{\mathrm{eff}}|F(z)|^{2}$, the power gain parameter $G=\Delta P / P$ given in Refs. 33 and 37 is the same as $\left[1 /\left(1+n_{\text {eff }}^{2}\right)\right](\bar{g} \times L)$ in our analysis of the small-signal approximation with neglecting the relaxation time effect. Note that if we neglect the contribution of the electron density modulation, the term of $\nabla \rho$ in Eq. (9) can be dropped and the power gain of the well-known previous analyses ${ }^{33,37}$ is exactly coincided with that derived in this paper.

\section{CONCLUSIONS}

We have investigated the stimulated emission of the CFEL in the small-signal gain and saturation regimes on the basis of the excitation of the EM waves by the current of the moving electron beam. The spatial growth rate of the gain is described using the modulations of the electron velocity and density by the EM field. We introduced the effect of the attenuation of the average velocity and the relaxation phenomenon into the dynamics formulas of the electron beam. In the small-signal approach, the interaction regions of the stimulated emission are categorized into the transition state and steady state basing on the interaction time is shorter and longer than the relaxation time, respectively. In the transition state, the growth rate is increased with the interaction time, and the relaxation time is of less importance. In the steady state, the growth rate becomes constant and is characterized by the relaxation time. In the saturation regime, we demonstrate a concrete reduction tendency of the growth rates due to the attenuation of the averaged velocity in the electron beam. In the small-signal approximation, we show that our results well match with those by other treatments based on completely different theoretical bases.

${ }^{1}$ J. E. Walsh and J. B. Murphy, IEEE J. Quantum Electron. 18, 1259 (1982).

${ }^{2}$ T. Shiozawa and H. Kondo, IEEE J. Quantum Electron. 23, 1633 (1987).

${ }^{3}$ F. Ciocci, G. Dattoli, A. Doria, G. P. Gallerano, G. Schettini, and A. Torre, Phys. Rev. A 36, 207 (1987).

${ }^{4}$ J. E. Walsh, T. C. Marshall, and S. P. Schlesinger, Phys. Fluids 20, 709 (1977).

${ }^{5}$ K. L. Felch, K. O. Busby, R. W. Layman, D. Kapilow, and J. E. Walsh, Appl. Phys. Lett. 38, 601 (1981).

${ }^{6}$ W. Main, R. Cherry, and E. Garate, IEEE Trans. Plasma Sci. 13, 524 (1985).

${ }^{7}$ S. M. Wiggins, D. A. Jaroszynski, B. W. J. McNeil, G. R. M. Robb, P. Aitken, A. D. R. Phelps, A. W. Cross, K. Ronald, N. S. Ginzburg, V. G. Shpak, M. I. Yalandin, S. A. Shunailov, and M. R. Ulmaskulov, Phys. Rev. Lett. 84, 2393 (2000).

${ }^{8}$ I. J. Owens and J. H. Brownell, Phys. Rev. E 67, 36611 (2003).

${ }^{9}$ S. C. Sharma and A. Bhasin, Phys. Plasmas 14, 53101 (2007).

${ }^{10}$ M.V. Kuzelev, Quantum Electron. 40, 83 (2010)

${ }^{11}$ E. P. Garate, R. Cook, P. Heim, R. Layman, and J. Walsh, J. Appl. Phys. 58, 627 (1985)

${ }^{12}$ E. P. Garate, C. H. Shaughnessy, and J. E. Walsh, IEEE J. Quantum Electron. 23, 1627 (1987).

${ }^{13}$ Y. U. Jeong, G. M. Kazakevitch, B. C. Lee, S. H. Park, and H. J. Cha, Nucl. Instrum. Methods. Phys. Res. A 528, 88 (2004).

${ }^{14}$ I. J. Owens and J. H. Brownell, J. Appl. Phys. 97, 104915 (2005). 
${ }^{15}$ D. Li, G. Huo, K. Imasak, and M. Asakawa, Nucl. Instrum. Methods. Phys. Res. A 606, 689 (2009).

${ }^{16}$ T. Taguchi and K. Mima, Nucl. Instrum. Methods. Phys. Res. A 331, 597 (1993).

${ }^{17}$ T. Taguchi and K. Mima, Nucl. Instrum. Methods. Phys. Res. A 341, 322 (1994).

${ }^{18}$ Y. Seo, E. H. Choi, and G. S. Cho, J. Phys. D: Appl. Phys. 33, 654 (2000).

${ }^{19}$ M. Yamada, IEEE J. Quantum Electron. 35, 147 (1999).

${ }^{20}$ Y. Kuwamura, M. Yamada, R. Okamoto, T. Kanai, and H. Fares, J. Appl. Phys. 104, 103105 (2008).

${ }^{21}$ H. Fares, M. Yamada, Y. Kuwamura, I. Matsumoto, and T. Kanai, IEEE J. Quantum Electron. 46, 981 (2010)

${ }^{22}$ H. Fares, M. Yamada, and Y. Kuwamura, Jpn. J. Appl. Phys. 49, 96402 (2010).

${ }^{23}$ A. Gover and A. Yariv, Appl. Phys. 16, 121 (1978).

${ }^{24}$ M. Asada and M. Yamada, J. Appl. Phys. 95, 5123 (2004).

${ }^{25} \mathrm{M}$. Reiser, Theory and Design of Charged Particle Beams (Wiley-VCH Verlag GmbH \& Co. KGaA, Weinheim, 2004), p. 461.
${ }^{26} \mathrm{~W}$. B. Thompson and J. Hubbard, Rev. Mod. Phys. 32, 714 (1960).

${ }^{27}$ S. Ichimm and M. N. Rosenbluth, Phys. Fluids 13, 2778 (1970).

${ }^{28}$ H. P. Freund, Phys. Rev. Lett. 65, 2993 (1990).

${ }^{29}$ D. S. Lemons and L. E. Thode, Phys. Rev. Lett. 56, 2684 (1986).

${ }^{30}$ J. S. Choi, E. G. Heob, B. H. Hong, and D. I. Choi, Nucl. Instrum. Methods. Phys. Res. A 375, 175 (1996)

${ }^{31}$ V. B. Asgekar and G. Dattoli, Opt. Commun. 255, 309 (2005).

${ }^{32} \mathrm{D}$. Li, G. Huo, K. Imasaki, M. Asakawa, and Y. Tsunawaki, Infrared Phys. Tech. 53, 204 (2010).

${ }^{33}$ T. Shiozawa, Classical Relativistic Electrodynamics (Springer, Berlin, 2004).

${ }^{34} \mathrm{~K}$. Horinouchi and T. Shiozawa, Electron. Comm. Jpn 2, 77, 12 (1994).

${ }^{35}$ I. de. la Fuente, P. J. M. van der Slot, and K. J. Boller, J. Appl. Phys. 100, 53108 (2006).

${ }^{36}$ J. D. Jackson, Classical Electrodynamics, 3rd ed. (John Wiley \& Sons, Inc, 1998), p. 246.

${ }^{37}$ A. Yariv and C. C. Shih, Opt. Commun. 24, 233 (1978). 